\title{
Freestanding Ultrathin Nanoparticle Membranes Assembled at Transient Liquid-Liquid Interfaces
}

\author{
Benjamin Le Ouay, Stefan Guldin, Zhi Luo, Sergio Allegri, and Francesco Stellacci*
}

\begin{abstract}
A synthetic route is presented for the realization of ultrathin freestanding nanoparticle membranes that are built of gold nanoparticles protected with trimethoxysilane-bearing ligands. The mechanism relies on interfacial assembly in an oil-water mixture. Upon shaking, nanoparticles are transported to the liquid-liquid interface of the oil droplets and form a network through the formation of Si-O-Si bridges. Reticulation of the nanoparticles during the dynamic process of droplet coalescence allows the formation of ultrathin membranes of only a few nanoparticle layers in thickness and square centimeters in dimension. The membranes can be manipulated, such as locally perforated, without causing their collapse. Furthermore they can be transferred onto solid or holey substrates. The synthetic route is compatible with a coassembly of dopants. As an example, membranes were doped with single walled carbon nanotubes, which resulted in a sizable increase of their electric conductivity.
\end{abstract}

as well as noncovalent ${ }^{[17,19]}$ reticulation or through the coassembly of the NPs with polymers, ${ }^{[29]} \mathrm{DNA}^{[30]}$ or peptides. ${ }^{[31]}$

Besides the classical thin-film assembly on a solid substrate (drop casting, ${ }^{[32]}$ spincoating, ${ }^{[27,33]}$ layer-by-layer deposition ${ }^{[8]}$ ) followed by a detachment step, interfacial assembly, and especially liquid-liquid assembly, is a very efficient way to obtain well-defined films or membranes ${ }^{[34-37]}$ (please note that in this context, we define as membrane any freestanding thin layer, regardless of selective mass-transport properties). These approaches rely on the possibility of NPs to adsorb and pack into a dense network at an interface. This can occur if the free energy of the nanoparticles bound at the interface is lower than that of the NPs in either of the two

\section{Introduction}

While nanoparticles (NPs) possess interesting properties, their assembly into organized suprastructures has been pursued to give access to novel physical and chemical behaviors. ${ }^{[1-5]}$ For example, the realization of large-scale films (i.e., quasi bidimensional assemblies) is a promising field. ${ }^{[6,7]}$ By removing the need of a support, realization of freestanding films can provide further advantages. ${ }^{[8,9]}$ For example, in catalytic ${ }^{[10-13]}$ or sensing devices, ${ }^{[14-16]}$ higher accessibility of the reagents to the NPs provides a functional advantage. In addition, the absence of a substrate allows mass transport through the assembly, an essential aspect for filtration applications. ${ }^{[17,18]}$ While lightweight and flexible, these systems possess high robustness, and can reversibly withstand significant deformations, ${ }^{[19,20]}$ allowing for the realization of accurate mechanical systems. ${ }^{[21,22]}$ The mechanical properties necessary to achieve a freestanding material can be obtained through covalent bonds, ${ }^{[23-27]}$ metal coordination ${ }^{[28]}$

Dr. B. Le Ouay, ${ }^{[+]}$Dr. S. Guldin, ${ }^{[+]}$Z. Luo, S. Allegri, Prof. F. Stellacci

Institute of Materials

Ecole Polytechnique Fédérale de Lausanne (EPFL)

1015, Lausanne, Switzerland

E-mail: francesco.stellacci@epfl.ch

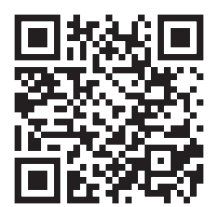

${ }^{[+]}$Present address: Department of Synthetic Chemistry and Biological Chemistry, Graduate School of Engineering, Kyoto University, Katsura, Nishikyo-ku, Kyoto 615-8510, Japan

${ }^{[++]}$Present address: Department of Chemical Engineering, University College London, Torrington Place, London WCIE 7JE, UK

DOI: 10.1002/admi.201600191 phases. ${ }^{[38-40]}$ The surface of NPs should thus possess specific wetting properties to guarantee a stable adsorption at the interface. Such behavior can be obtained using nanoparticles with a Janus-type anisotropic surface functionalization ${ }^{[41]}$ or having systems that will spontaneously break their symmetry in situ. ${ }^{[42]}$ This can also be obtained by carefully modifying locally the properties of one of the solvents to create a layer of intermediate characteristics, for instance, by injecting locally a compatibilizing reagent such as ethanol ${ }^{[43]}$ or by applying an electric field. ${ }^{[4]}$

Hereby, we propose an innovative approach to achieve the formation of ultrathin free-standing and mechanically resilient membranes. The membranes are constituted of gold nanoparticles (AuNPs) that are reticulated by bridging mercaptopropyltrimethoxysilane (MPTMS) ligands which can cross-link through the formation of $\mathrm{Si}-\mathrm{O}-\mathrm{Si}$ bonds. Our approach relies on the formation of an unstable oil-in-water emulsion, in which AuNPs assemble at the surface of the oil droplets. Upon coalescence of the droplets, the decreasing available surface causes the AuNPs to close-pack and cross-link via the MPTMS ligands, forming an ultrathin membrane that remains freestanding in the water-phase. This dynamic interfacial assembly guarantees the presence of only a few NP-layers, with monolayer-thin domains easily obtained over several square-millimeters. Furthermore, the membranes can then be transferred onto solid or holey support substrates. This synthetic route is compatible with the presence of functional dopants that are physically immobilized in the AuNPs network, making this a promising way to form functional membranes by combining targeted properties through co-assembly. Since the preparation procedure involves the intimate contact of the water and oil phases 
before cross-linking, this could be used to associate components dispersed in water with AuNPs dispersed in the organic phase, a feature difficult to achieve with systems at equilibrium. We demonstrate this for percolating carbon nanotubes showing an electrically conductive membrane.

\section{Results and Interpretation}

\subsection{Nanoparticles Synthesis}

Nanoparticles were prepared following a methodology adapted from Zheng et al., ${ }^{[45]}$ using MPTMS as ligand. In this synthetic route, the use of a gold (I) complex and of a mild reducing agent allows a good control over the size distribution of the sample. Furthermore, this reaction can be performed in a wide variety of solvents systems, and allows the preparation of a broad range of ligand-protected nanoparticles, including mixedligands. ${ }^{[46,47]}$ In the present study, the mean diameter of the obtained AuNPs was $5.2( \pm 0.9) \mathrm{nm}$, as measured by Transmission Electron Microscopy (TEM) (Figure S1, Supporting Information). We chose to use a solvent mixture (toluene/ethanol $4: 1 \mathrm{v} / \mathrm{v}$ ) in order to allow a good solubility of all reagents and products during the synthesis. Anhydrous solvents were used in order to limit the hydrolysis (and subsequent condensation) reaction of the MPTMS ligands. After synthesis, the solvent was exchanged to dichloromethane (DCM), in which the methoxyprotected nanoparticles were readily soluble. DCM is also ideal for the interfacial assembly process, as it has a density above 1 , and possesses a slight miscibility with water $\left(16 \mathrm{~g} \mathrm{~L}^{-1}\right)^{[48]}$ that leads to a more efficient phase transfer of the nanoparticles.

\subsection{Membrane Preparation}

Membranes were prepared by the addition of a mildly acidic $(\mathrm{pH}=2)$ or basic $(\mathrm{pH}=14)$ water solution to a dilute AuNPs suspension in DCM and subsequent manual shaking. The formation of the membrane is shown in Figure 1A-D. Given its dynamic nature, we highly recommend the reader to see videos of these processes and refer to S2 and S3 in the Supporting Information. The system was initially constituted of nanoparticles dispersed in the DCM phase with a AuNPs-free aqueous phase situated above (Figure 1A). After vigorous shaking, we observed the formation of an unstable oil-in-water emulsion that coalesced within seconds (Figure 1B,C). During the coalescence, the AuNPs assembled around the droplets of DCM and formed a membrane. After full coalescence and sedimentation of the DCM to the bottom of the vial, the membrane remained free-standing in the water phase, and was attached to both the air/water and water/DCM interfaces. This resulted in the formation of a continuous 2D membrane that spanned vertically across the full heights of the water phase, as shown in Figure 1D. Once the membrane was formed and its surface stabilized, unbound AuNPs were released in the DCM phase, as evidenced by the presence of a tenuous flow of ruby-red color in the DCM phase on Figure 1D. This phenomenon may contribute to the control of the membrane thickness, as excess AuNPs tended to be released instead of building an extra layer.
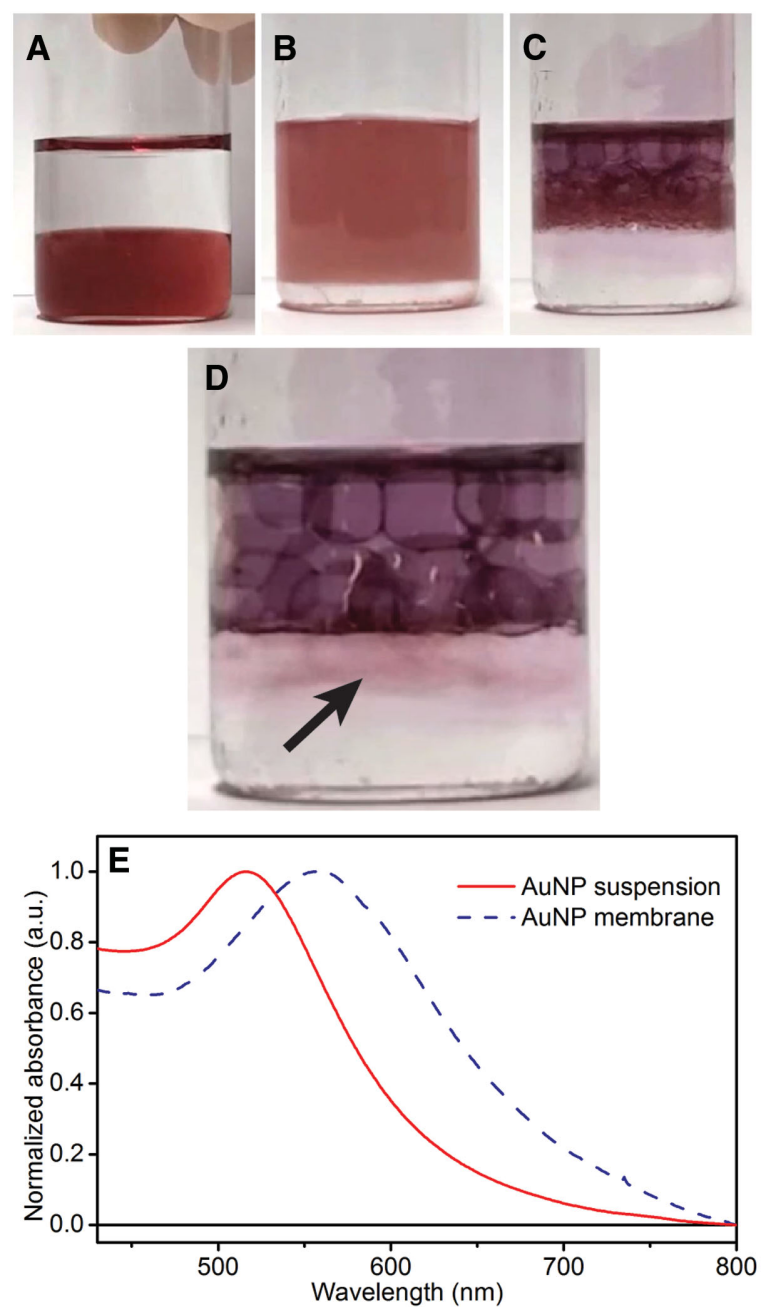

Figure 1. A-D) Photographs of an AuNPs in DCM-water system before shaking $(A)$ and at different stages of the coalescence of DCM (B-D). The arrow in (D) indicates a release of some AuNPs that were not integrated in the membrane. E) Normalized absorbance spectra of AuNPs in suspension (plain) and as a membrane suspended in water (dashed line).

Interestingly, while unperturbed membranes were stable for several hours, vigorous shaking of the system led momentarily to a dispersion of the nanoparticles that retrieved a ruby-red color for a few seconds. Video S2 in the Supporting Information shows how a new and intact membrane was then formed as the DCM coalesced again. The cross-linking of the AuNPs can be followed by the change in color of the AuNPs (Figure 1E). Before shaking as well as in the early stages of the coalescence, the solution had a bright ruby red color that is attributable to the plasmon absorption band of dispersed AuNPs. Upon coalescence, the nanoparticles reached a critical surface concentration that forced them into close-packing. This resulted in an abrupt color change toward the violet. Accordingly the characteristic absorbance spectra broadened and the position of the maximum shifted from 517 to $560 \mathrm{~nm}$, reasonably due to the plasmon coupling of vicinal nanoparticles. ${ }^{[49]}$

Based on our observations, we can speculate the following mechanism, represented on Figure 2. After vigorous shaking, we form an unstable DCM-in-water emulsion that exhibits a 

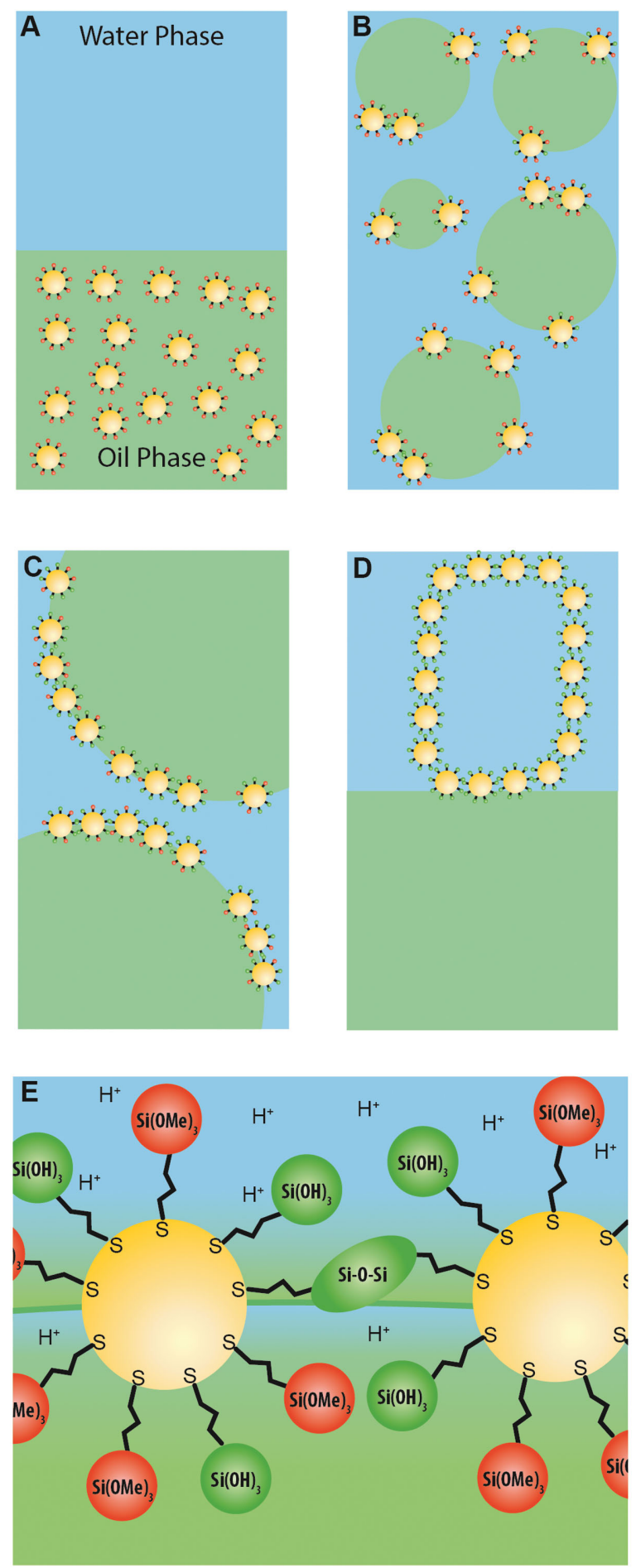

Figure 2. Scheme of the anticipated mechanism for the formation of AuNP membranes. A) AuNPs are initially in the oil phase, with nonhydrolyzed MPTMS. B) Upon shaking, small DCM droplets in water are formed, and partially hydrolyzed AuNPs assemble at the oil-water interface. C) As the droplets coalesce, the interfacial surface decreases, and induces close-packing of the AuNPs. D) This results in a membrane that remains in the water phase, as the DCM retracts by gravity. E) Mechanical stability of the membrane is assured by condensation of the MPTMS ligands around the nanoparticles while they are at the interface. high interfacial area. Under acidic or basic conditions, a fraction of the methoxy groups of MPTMS ligands are cleaved, providing reactive $\mathrm{Si}-\mathrm{OH}$ moeities. Partially unprotected nanoparticles have intermediate solubility properties and are transported to the DCM/water interface to form a local 2D assembly. ${ }^{[50]}$ On this interface, condensation of $\mathrm{Si}-\mathrm{OH}$ groups occurs between adjacent AuNPs to form $\mathrm{Si}-\mathrm{O}-\mathrm{Si}$ bridges.${ }^{[1]}$ As the DCM droplets coalesce and sediment toward the bottom of the vial by gravity, the interfacial area decreases. AuNP rafts present on this interface are therefore brought in contact and bind with one another to form a 2D-network. This results in the formation of a continuous membrane that is spanning over several square centimeters.

The success of the nanoparticles assembly was dependent on several parameters of chemical as well as geometrical nature. The role of crucial parameters for the formation of membranes is summarized in Table 1. We refer to a video showing membrane formation under non-optimized conditions in the Supporting Information (Video S4).

While partial miscibility of the organic solvent with water seemed crucial to ensure hydrolysis, the relative density did not play a role. Membranes could also be formed when using toluene, that has a lower density than water. The use of DCM was, however, preferred, as the AuNPs membrane was then formed in the top phase and thus more accessible. Importantly, geometric factors had to be considered. Assuming dense packing, the maximum coverable area is proportional to the quantity of AuNPs. This area has to be compared to the dimensions of the water phase (height and vial diameter). Accordingly, membranes were not formed when the quantity of AuNPs was insufficient (or, equivalently, if the volume of the aqueous phase was too high). On the opposite case, high quantities of AuNPs led to the formation of a reticulated foam with several smallsized cells to accommodate the large coverage surface. A geometrical calculation, considering hexagonal 2D close-packed nanoparticles with a packing density of 0.907 , indicates that 1 mg of $5.2 \mathrm{~nm}$ sized AuNPs can cover $165 \mathrm{~cm}^{2}$ (see Supporting Information). The volume of the aqueous phase was chosen accordingly so that the selected amount of AuNPs could form a monolayer in the vials that were used.

The $\mathrm{pH}$ of the water phase is another important factor, as it was found to influence the kinetics of hydrolysis and thus the reticulation of the ligands. The $\mathrm{pH}$-dependent reactivity of silicon alkoxides is well-known, being the staple of silica solgel chemistry. ${ }^{[1]}$ Two related parameters were crucial for the preparation of membranes. The first one was the speed of reaction compared to the one of coalescence of the oil droplets, the second one the stability of the bridging $\mathrm{Si}-\mathrm{O}-\mathrm{Si}$ bonds in the medium. To fulfill the first condition, the water phase had to be either acidic enough $(\mathrm{pH} \leq 2)$ or basic enough $(\mathrm{pH} \geq 12)$ in order to obtain a hydrolysis rate of the methoxy groups that was sufficient to create a network during the few seconds of coalescence. Furthermore, very acidic solutions $\left(\left[\mathrm{H}_{3} \mathrm{O}^{+}\right]>1 \mathrm{~mol} \mathrm{~L}^{-1}\right)$ led to the formation of aggregates instead of a homogeneous membrane. One reason could be that high acid concentrations led to a significant hydrolysis in the (hydrated) DCM phase before the interfacial assembly, and thus to a reticulation of the AuNPs in solution to form 3D-aggregates. In addition, the reaction conditions had to be chosen so that hydrolysis of $\mathrm{Si}-\mathrm{O}-\mathrm{Si}$ 
Table 1. Influence of crucial experimental parameters on the formation of AuNP membranes. Optimized conditions are: $6 \mathrm{~mL}$ of MPTMSprotected AuNPs suspension ( $0.05 \mathrm{mg} \mathrm{mL}^{-1} \mathrm{Au}$ ) in dichloromethane, in contact with $7.5 \mathrm{~mL}$ of an aqueous $\mathrm{HCl}$ solution $\left(0.2 \mathrm{~mol} \mathrm{~L}^{-1}\right)$. The membrane is obtained by shaking. The various processing tests were performed following the alteration stated above under otherwise identical experimental conditions.

\begin{tabular}{|c|c|c|}
\hline Conditions & $\begin{array}{l}\text { Altered experimental } \\
\text { parameter }\end{array}$ & Observations \\
\hline $\begin{array}{l}\text { Optimized acidic } \\
\text { conditions }\end{array}$ & N.A. & $\begin{array}{l}\text { Freestanding membrane } \\
\text { spread vertically in the } \\
\text { aqueous phase }\end{array}$ \\
\hline $\begin{array}{l}\text { Alkaline route followed by } \\
\text { neutralization (optimized } \\
\text { alkaline conditions) }\end{array}$ & $\begin{array}{c}\text { Alkaline route followed by } \\
\text { neutralization with } \mathrm{HCl} \\
(7 \mathrm{~mol} / \mathrm{l}) \text { and shaking }\end{array}$ & $\begin{array}{l}\text { Stable freestanding mem- } \\
\text { brane was formed. }\end{array}$ \\
\hline $\begin{array}{l}\text { Nonreticulating } \\
\text { nanoparticles }\end{array}$ & $\begin{array}{c}\text { Octanethiol-protected } \\
\text { AuNPs }\end{array}$ & No phase transfer \\
\hline Absence of shaking & $\begin{array}{l}\text { No shaking. Reaction is } \\
\text { carried out overnight. }\end{array}$ & $\begin{array}{l}\text { Partial transport of the } \\
\text { AuNPs at the liquid-liquid } \\
\text { interface. Aggregation of } \\
\text { the nanoparticles in DCM }\end{array}$ \\
\hline $\begin{array}{l}\text { Higher volume of the } \\
\text { aqueous phase }\end{array}$ & $15 \mathrm{~mL} \mathrm{HCl}\left(0.1 \mathrm{~mol} \mathrm{~L}^{-1}\right)$ & $\begin{array}{l}\text { Membrane did not spread } \\
\text { in the aqueous phase. } \\
\text { Air bubbles trapped under } \\
\text { the membrane could lift } \\
\text { the membrane locally, } \\
\text { forming "veils". }\end{array}$ \\
\hline Higher NP concentration & $0.5 \mathrm{mg} \mathrm{mL}^{-1}$ AuNPs & $\begin{array}{l}\text { Foam stabilized by AuNPs } \\
\text { formed in the water phase }\end{array}$ \\
\hline Higher acid concentration & $1 \mathrm{~mol} \mathrm{~L}^{-1} \mathrm{HCl}$ & $\begin{array}{l}\text { Membrane was } \\
\text { formed but presented } \\
\text { inhomogeneities. }\end{array}$ \\
\hline Lower acid concentration & $0.001 \mathrm{~mol} \mathrm{~L}^{-1} \mathrm{HCl}$ & $\begin{array}{l}\text { No membrane formation } \\
\text { over short time }\end{array}$ \\
\hline Mild alkaline conditions & $0.001 \mathrm{~mol} \mathrm{~L}^{-1} \mathrm{NaOH}$ & No membrane formation \\
\hline Alkaline conditions & $\begin{array}{c}1 \mathrm{~mol} \mathrm{~L}^{-1} \mathrm{NaOH}+50 \\
\mathrm{mmol} \mathrm{L}^{-1} \mathrm{Na}_{2} \mathrm{CO}_{3}\end{array}$ & $\begin{array}{l}\text { Membrane formed then } \\
\text { collapsed instantly. Iden- } \\
\text { tical behavior in absence of } \\
\qquad \mathrm{Na}_{2} \mathrm{CO}_{3}\end{array}$ \\
\hline
\end{tabular}

bridges remained slow enough to allow the AuNPs to assemble into a continuous 2D membrane. This forbade extremely low, or high $\mathrm{pH}(\mathrm{pH}<0$ and $\mathrm{pH}>10) \cdot{ }^{[51]}$ Once again, the conditions described in the Experimental Section were optimized to obtain reproducible and homogeneous AuNP membranes. In the acidic range, these two regimes were compatible. We developed one route for the preparation of membranes using mildly acidic $(\mathrm{pH}=2)$ conditions as it catalyzed hydrolysis in the course of DCM coalescence, while avoiding the formation of aggregates. In the alkaline range, domain of stability of the $\mathrm{Si}-\mathrm{O}-\mathrm{Si}$ bridges and domains of sufficient hydrolysis rate did not overlap, so that stable AuNPs membranes could not be formed in basic conditions. It was however possible to form the membranes through alkaline hydrolysis in a two step process. The first step consisted of hydrolyzing MPTMS under highly basic conditions $\left(1 \mathrm{~mol} \mathrm{~L}^{-1} \mathrm{NaOH}\right)$ in the presence of a buffering salt $\left(0.05 \mathrm{~mol} \mathrm{~L}^{-1} \mathrm{NaHCO}_{3}\right)$. In this case, the high lability of the $\mathrm{Si}-\mathrm{O}-\mathrm{Si}$ bond in an alkaline medium led to a lower mechanical stability and thus to a collapse and redispersion of the membrane shortly after its formation. In a second step, the base was neutralized after partial hydrolysis by addition of a precise volume of concentrated acid $\left(7 \mathrm{~mol} \mathrm{~L}^{-1} \mathrm{HCl}\right)$, in order to significantly slow down the hydrolysis of the reticulating $\mathrm{Si}-\mathrm{O}-$ Si network. The presence of the buffering salt facilitated the control of the resulting neutral $\mathrm{pH}$ by making it less sensitive to small variations of the acid volume. A video of a membrane formation following this route can be seen in the Supporting Information (Video S3).

\subsection{Membrane Deposition}

Once formed, membranes spread vertically over the height of the water phase. While their mechanical resistance was sufficient to support their own weight, their surface could be easily manipulated and perforated with a sharp object, such as a needle (Video S2, Supporting Information). Such action caused localized holes, but did not lead to the collapse of the full membrane, indicating that the assembly was not stabilized by surface tension.

Samples for TEM observation were prepared by immersing a grid into the solution and bringing it in contact with the membrane. We used either carbon-coated or bare copper grids having both a span of $42 \mu \mathrm{m}$. As depicted in Figure 3B,C, the membranes were constituted of a dense-packed assembly of AuNPs. The network presents relatively few packing faults, a remarkable feature for a system assembled through a dynamic process within seconds. Images obtained at a lower magnification (Figure 3A) indicate that the membrane spread evenly over full squares of the TEM grids, with relatively few folding defects or ruptures. Membranes were also deposited onto bare copper TEM grids. In absence of a support for the membrane, we were unable to observe an intact membrane spread over the full hole of the grid $(42 \times 42 \mu \mathrm{m})$. However, we were able to observe domains where the membrane was pinned to corners of the squares. In such areas, freestanding membranes spread without a support, over distance of several tens of micrometers (Figure 3D-F). In this case, the membrane was constituted of more than one monolayer, as indicated by the overlapping of nanoparticles (Figure 3F). Please note that a bias in the experiment could exist, as thicker membranes are more robust and thus more likely to be observable by TEM.

It was also possible to coat centimeter-sized substrates using the same deposition technique, as shown for cellulose-ester in Figure 4A. This substrate exhibits a sponge-like structure with walls having a thickness of around $300 \mathrm{~nm}$ (Figure 4B). SEMimaging of the substrate indicated a conformal deposition on the surface of the substrate (Figure 4C). To some extend, a coarsening of the morphological features was observed after coating. This can be explained by the spreading over several edges of the sponge texture, resulting in a smoother surface.

Long-distance homogeneity of the membrane was observed by optical microscopy and integrated microspectroscopy. Our setup allowed spectroscopy of the membrane supported on carbon with a collection spot size of $7 \mu \mathrm{m}$. This spatial resolution allowed to probe different meshes on the TEM grid as well 

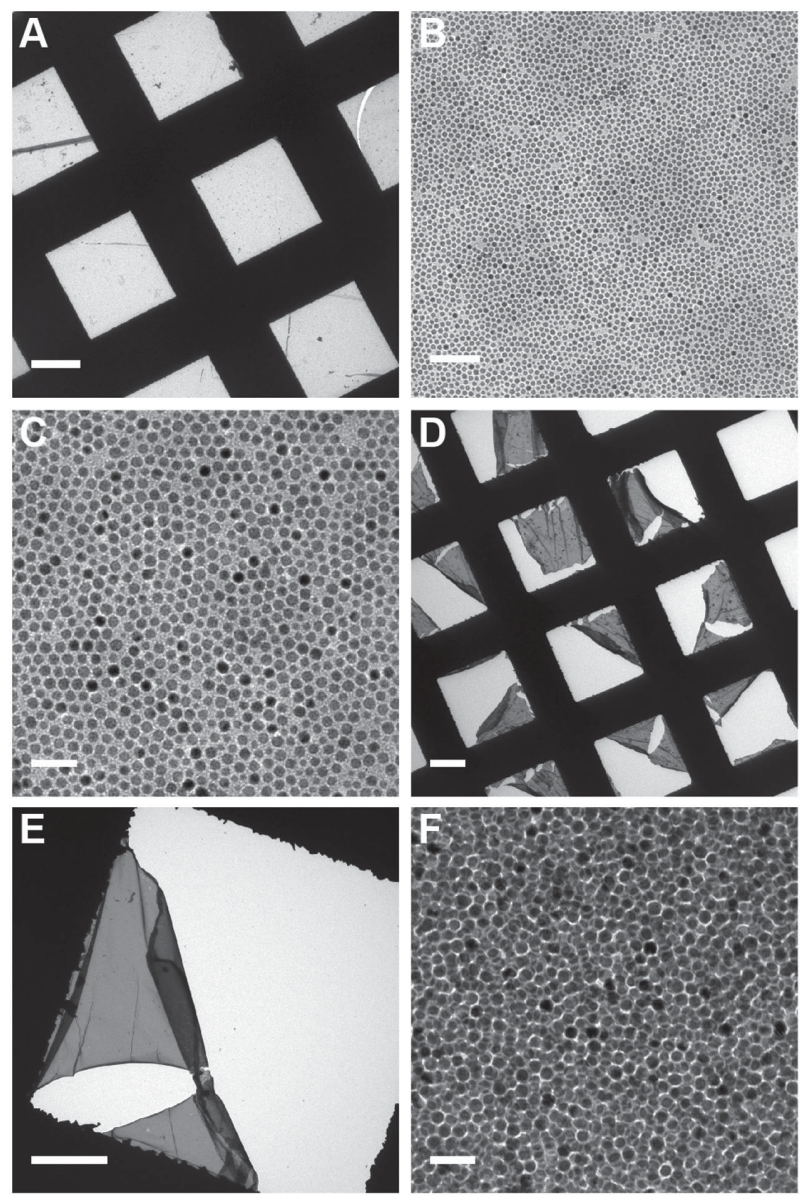

Figure 3. TEM images of an AuNP membrane supported on a carbon film $A-C)$, and freestanding $D-F)$. The scale bars represent (A) $20 \mu \mathrm{m}$; (B) $50 \mathrm{~nm}$; (C) $20 \mathrm{~nm}$; (D) $20 \mu \mathrm{m}$; (E) $10 \mu \mathrm{m}$, (F) $20 \mathrm{~nm}$.

as several points on the same mesh. Typically, the interior of the mesh was found to be homogeneous in color, and absorbance spectra were similar in shape and intensity (Figure S6, Supporting Information). The spectra exhibited a broad absorption band. This band can be attributed to a broadened and redshifted plasmon band resulting from the close-packing of nanoparticles. The homogeneity in spectral shape and intensity is consistent with the presence of a homogeneous membrane of nanoparticles on top of the TEM grid. Furthermore, the film had the same thickness in the different points of the grid, probably constituted of a dense monolayer, as indicated by TEM (Figure 3).

We were able to observe a domain where the nanoparticle membrane formed buckles. In this domain, the presence of homogeneous darker zone was attributed to extra nanoparticles layers on top of the base membrane. The collection spots are indicated as dotted white circles in Figure 5A, and the respective associated spectra after subtraction of the carbon support background spectrum are represented in Figure 5B. Intensity of the absorbance varied depending on the point of collection. Absorbance values were normalized to the one of the base membrane by dividing the value by 0.085 . For each measurement point, the maximal normalized absorbance was close to an integer value. As observed in the case of graphene, ${ }^{[52]}$ this is in agreement with the stacking of several layers of the AuNP membrane, each one contributing equally to the material absorbance.

\subsection{CNT-Doped AuNP Membranes}

Having proven the possibility to form thin membranes at the transient water/DCM assembly, we studied the incorporation of dopants with complementary function. This approach relies on the phenomena that most types of nanomaterial are transported, at least temporarily, to the interface and may therefore coassemble in the membrane formation process. ${ }^{[33,54]}$ In order to provide electronic conduction properties to the membrane, we incorporated single-walled carbon nanotubes (SWCNTs), following the acidic route to form the membrane. Provided the loading rate was sufficiently low (below $20 \mathrm{wt} \%$ ), the addition of SWCNTs did not perturb the formation of the membrane. Incorporation of the SWCNTs can be confirmed by the darker color of the membrane along with their depletion from the water phase. TEM-imaging indicated that the membranes formed in presence of SWCNTs were typically also constituted of a single AuNPs monolayer, in which the SWCNTs were integrated (Figure 6A,B). Considering that we used nonfunctionalized nanotubes dispersed in water with a surfactant (Triton $\mathrm{X}-100)$, chemical bonding between SWCNTs and MPTMS
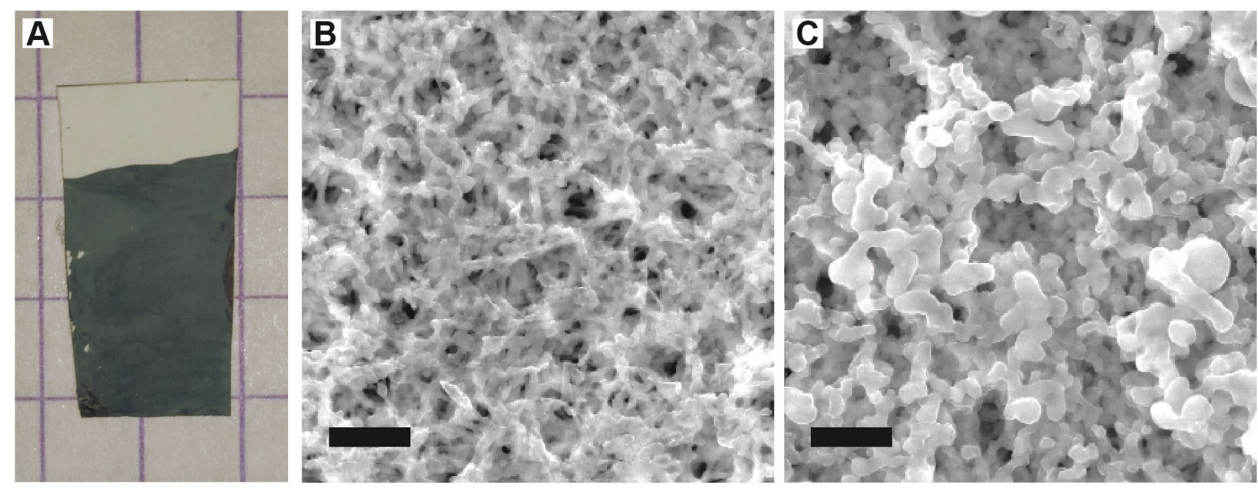

Figure 4. A) Photograph of a cellulose ester substrate coated with an AuNP membrane. B,C) SEM images of the cellulose ester substrate before (B) and after (C) coating. The scale bars represent $2 \mu \mathrm{m}$. 

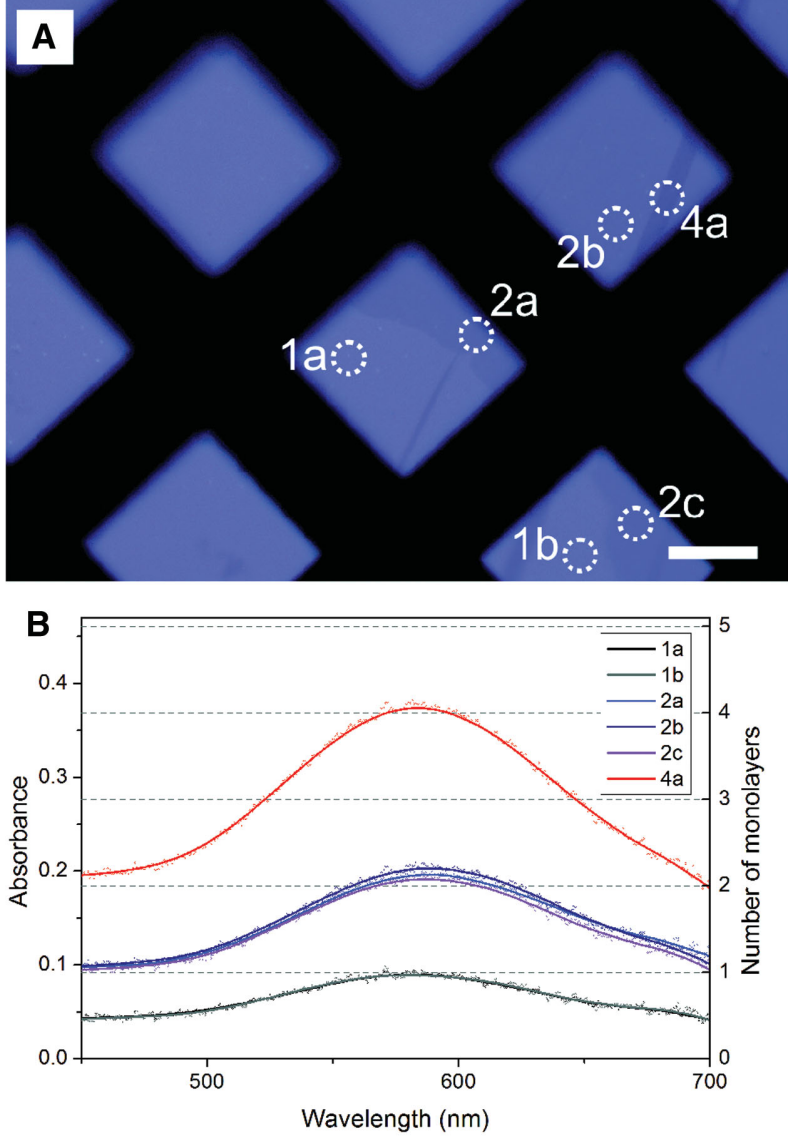

Figure 5. Optical microscope views A) of an AuNP membrane supported on a carbon film. The dashed circles correspond to the zone where microspectroscopic measurements were performed. B) Plot of corresponding spectra between 450 and $700 \mathrm{~nm}$ after subtraction of the carbon support background spectrum. The number indicates the anticipated number of monolayers, while the letter identifies the spectrum. The scale bar represents $20 \mu \mathrm{m}$.

is unlikely, and thus we anticipate that the immobilization was obtained through physical bonding or encapsulation of the SWCNTs by the AuNPs. Resistivity of the SWCNTs was measured at $12 \mathrm{k} \Omega \mathrm{sq}^{-1}$ (2-points set-up) for a doping-rate of $11.7 \mathrm{wt} \%$, while undoped films exhibited no measurable conductivity
$\left(R>20 \mathrm{M} \Omega \mathrm{sq}^{-1}\right)$, as expected for monolayers of weakly coupled AuNPs. ${ }^{[55]}$ Taking into account the very low thickness of the membranes ( $<10 \mathrm{~nm}$ ), this corresponds to a conductivity of $>83 \mathrm{~S} \mathrm{~cm}^{-1}$. While not as high as for pure CNTs films, this value is comparable with other ultrathin films of CNT-doped composites. ${ }^{[56]}$

In the case of higher doping-rate ( $25 \mathrm{wt} \%$ and above) membranes were much less stable and collapsed quickly. This was attributed to the fact that the SWCNTs could themselves cover a very high surface, forming a nonreticulated network. Furthermore, the presence of several long objects at the surface of the DCM limited the migration of the nanoparticles during the coalescence of the droplets, preventing the formation of a densely reticulated AuNPs network (Figure 6C). However, even in the case of excessive doping-rate, the process still allowed the preparation of membranes that could be deposited on substrates. While less suitable for filtration application, such materials may find applications in catalysis or sensing, given the very high accessibility of the NPs in intimate contact with the SWCNTs, along with their high conductivity.

\section{Conclusion}

We described a facile procedure for the realization of freestanding membranes that are constituted of monolayers of AuNPs. The process is based on the self-assembly of AuNPs at an instable DCM-water interface, followed by the reticulation of AuNPs and immobilization of the network by the formation of Si-O-Si bridges that arise from the ligand shell of the AuNPs. The phenomenon is dynamic and occurs upon coalescence of the DCM droplets, as AuNPs are brought to higher proximity. Since the formation arises at the boundary between two phases, the process leads to well-defined monolayers, with a thickness below $5 \mathrm{~nm}$. AuNP membranes can easily be transferred onto substrates, or spread over holes up to several dozen of micrometers. Furthermore, the AuNPs coassemble with other type of nanomaterials, and immobilize them in the membrane, as demonstrated here with SWCNTs. This approach may be extended to other types of nanoparticles, either as the base of the membrane or as the functional charge, and other reticulation reactions may also be used to accommodate more fragile systems. This approach represents a versatile route for
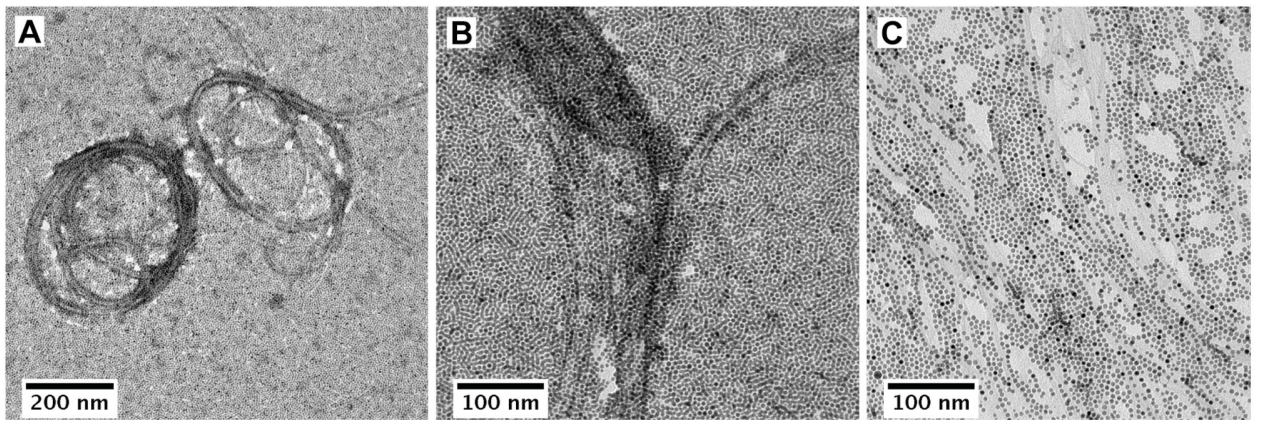

Figure 6. A,B) TEM images of a SWCNT doped (11.7 wt\%) AuNP membrane. At this doping rate, SWCNTs are well integrated in the membrane. C) TEM images at a higher doping rate $(28 \mathrm{wt} \%)$. The AuNPs formed low dimension rafts but did not span to a continuous membrane, thus preventing the fusion of SWCNTs. 
the realization of ultrathin functional membranes and has the potential to be applied for the realization of a wide range of applications.

\section{Experimental Section}

Synthesis of MPTMS-Protected AuNPs: AuClP(Ph) ${ }_{3} \quad(0.2 \mathrm{mmol})$ and MPTMS $(0.2 \mathrm{mmol})$ were dissolved in a mixture of anhydrous toluene $(80 \mathrm{~mL})$ and anhydrous ethanol $(20 \mathrm{~mL})$. The solution was subsequently heated to reflux under a circulation of argon. Once the temperature stabilized, $1.3 \mathrm{mmol}$ of borane tert-butylamine complex was dissolved in $15 \mathrm{~mL}$ anhydrous ethanol and then rapidly added to the solution. The solution, initially colorless, turned progressively brown then red as AuNPs formed. The reaction was maintained under reflux and argon circulation for $1 \mathrm{~h}$ and then let to cool down. The solvent was subsequently evaporated under reduced pressure, until the volume reached $20 \mathrm{~mL}$, and the nanoparticles were then precipitated by adding $80 \mathrm{~mL}$ of anhydrous hexane. The NPs were washed three times by centrifugation in hexane and then dispersed in $40 \mathrm{~mL}$ anhydrous DCM. Finally, the suspension was centrifuged once, and the supernatant containing dispersed nanoparticles was stored under argon. Nanoparticles suspensions stored under anhydrous conditions were stable over several weeks.

Formation of the Gold Nanoparticle Membranes: Au-MPTMS stock suspension was diluted in DCM to reach an absorbance of 0.6 at $517 \mathrm{~nm}$ (this corresponds to a concentration of $0.05 \mathrm{mg} \mathrm{mL}^{-1}$ ). Two routes were designed to obtain membranes, one in acidic and the other one in alkaline conditions. $7.5 \mathrm{~mL}^{-1}$ of Au-MPTMS suspension and $6 \mathrm{~mL}$ of an aqueous solution containing either $\mathrm{HCl}\left(0.01 \mathrm{~mol} \mathrm{~L}^{-1}\right)$ or $\left.\mathrm{NaOH}(1 \mathrm{~mol} \mathrm{~L})^{-1}\right)$ and $\mathrm{NaHCO}_{3}\left(0.05 \mathrm{~mol} \mathrm{~L}^{-1}\right)$ were introduced in a narrow cylindrical vial $(2.8 \mathrm{~cm}$ inner diameter $)$. The system was vigorously shaken manually until obtaining a (temporary) homogeneous dispersion. As the DCM phase, depleted of its AuNPs, coalesced and moved to the bottom of the vial, the AuNP membrane formed and remained freestanding in solution. In the alkaline route, the solution was neutralized after shaking with a precise volume of concentrated $\mathrm{HCl}$ solution $\left(7 \mathrm{~mol} \mathrm{~L}^{-1}\right)$. A new membrane could be formed subsequently by repeated shaking. The exact volume of acid needed to reach $\mathrm{pH}=7$ was determined within a $1 \mu \mathrm{L}$ range with test solutions prior to the addition.

SWCNT-Doped Membranes: SWCNT (Sigma-Aldrich, aqueous ink $\left.\left(1.00 \mathrm{mg} \mathrm{mL}^{-1}\right)\right)$ were diluted in $\mathrm{HCl}\left(0.01 \mathrm{~mol} \mathrm{~L}^{-1}\right)$ to a concentration

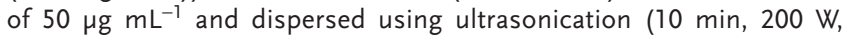
$20 \mathrm{kHz}$, solution maintained at $0^{\circ} \mathrm{C}$ ). Membranes were prepared following the acidic route described earlier, by replacing a fraction of the $\mathrm{HCl}$ solution with SWCNT-HCl. For electrical measurements, membranes were deposited onto cellulose ester filters, then cut as $1 \mathrm{~cm}$ bands. Resistances were measured using a Hewlett Packard HP $4145 \mathrm{~B}$ semiconductor analyzer, between two electrodes separated by $1 \mathrm{~cm}$.

Transmission Electron Microscopy: Samples were prepared by passing TEM grids (400 mesh, bare or carbon-coated, Electron Microscopy Sciences) through the AuNP membrane. The observations were performed on a Philips CM-12 microscope. Electron source was a $\mathrm{LaB}_{6}$ crystal, operating under an accelerating tension of $100 \mathrm{kV}$.

Scanning Electron Microscopy: Observations were performed on a FEI XLF30-FEG, under an acceleration tension of $27 \mathrm{kV}$. Samples were attached on aluminum holders using carbon double-face tape, and carbon was evaporated on their surface prior to observation.

Microspectroscopic Measurements: Spectroscopic absorption measurements were carried out on an Olympus BX61 microscope with 50x magnifying objective (LMPlanFl, NA 0.5 ) and detected through a microspectroscopy port with a $50 \mu \mathrm{m}$ fiber and an Ocean Optics QE 65000 spectrometer. This resulted in a collection spot size of around $7 \mu \mathrm{m}$. The absorbance of the supporting carbon film was obtained by taking a reference spectrum without AuNP membrane.

\section{Supporting Information}

Supporting Information is available from the Wiley Online Library or from the author.

\section{Acknowledgements}

B.L.O. acknowledges support from the CTI (13945.2), in partnership with Dentsply-Maillefer (Baillaigues, Switzerland). S.G. is grateful for support by the German National Academy of Sciences Leopoldina, Fellowship LPDS2012-13. F.S. is greatful for the support from the Swiss National Science Foundation.

Received: March 8, 2016

Revised: April 7, 2016

Published online: May 25, 2016

[1] Y. Min, M. Akbulut, K. Kristiansen, Y. Golan, J. Israelachvili, Nat. Mater. 2008, 7, 527

[2] M. Grzelczak, J. Vermant, E. M. Furst, L. M. Liz-Marzán, ACS Nano 2010, 4, 3591

[3] Z. Nie, A. Petukhova, E. Kumacheva, Nat. Nano 2010, 5, 15.

[4] Y. Gao, Z. Tang, Small 2011, 7, 2133.

[5] J. B. Edel, A. A. Kornyshev, A. R. Kucernak, M. Urbakh, Chem. Soc. Rev. 2016, 45, 1581.

[6] A. N. Shipway, E. Katz, I. Willner, ChemPhysChem 2000, $1,18$.

[7] D. Lee, M. F. Rubner, R. E. Cohen, Nano Lett. 2006, 6, 2305.

[8] C. Jiang, V. V. Tsukruk, Adv. Mater. 2006, 18, 829.

[9] W. Cheng, M. J. Campolongo, S. J. Tan, D. Luo, Nano Today 2009, 4, 482.

[10] X. Huang, S. Tang, X. Mu, Y. Dai, G. Chen, Z. Zhou, F. Ruan, Z. Yang, N. Zheng, Nat. Nano 2011, 6, 28.

[11] I. Hatay, P. Y. Ge, H. Vrubel, X. Hu, H. H. Girault, Energy Environ. Sci. 2011, 4, 4246.

[12] X. Bian, M. D. Scanlon, S. Wang, L. Liao, Y. Tang, B. Liu, H. H. Girault, Chem. Sci. 2013, 4, 3432.

[13] E. Smirnov, P. Peljo, M. D. Scanlon, H. H. Girault, ACS Nano 2015, 9, 6565 .

[14] C. Jiang, S. Markutsya, Y. Pikus, V. V. Tsukruk, Nat. Mater. 2004, 3, 721.

[15] M. Hojeij, N. Younan, L. Ribeaucourt, H. H. Girault, Nanoscale 2010, 2, 1665

[16] Q. Yu, H. Huang, X. Peng, Z. Ye, Nanoscale 2011, 3, 3868.

[17] J. He, X.-M. Lin, H. Chan, L. Vukovi, P. Král, H. M. Jaeger, Nano Lett. 2011, 11, 2430.

[18] S. Rao, K. J. Si, L. W. Yap, Y. Xiang, W. Cheng, ACS Nano 2015, 9, 11218.

[19] K. E. Mueggenburg, X.-M. Lin, R. H. Goldsmith, H. M. Jaeger, Nat. Mater. 2007, 6, 656 .

[20] H. Schlicke, E. W. Leib, A. Petrov, J. H. Schröder, T. Vossmeyer, J. Phys. Chem. C 2014, 118, 4386.

[21] H. Schlicke, D. Battista, S. Kunze, C. J. Schröter, M. Eich, T. Vossmeyer, ACS Appl. Mater. Interfaces 2015, 7, 15123.

[22] H. Schlicke, M. Rebber, S. Kunze, T. Vossmeyer, Nanoscale 2016, 8, 183.

[23] Y. Lin, H. Skaff, A. Böker, A. D. Dinsmore, T. Emrick, T. P. Russell, J. Am. Chem. Soc. 2003, 125, 12690.

[24] P. Selvakannan, P. S. Kumar, A. S. More, R. D. Shingte, P. P. Wadgaonkar, M. Sastry, Adv. Mater. 2004, 16, 966.

[25] J. T. Russell, Y. Lin, A. Böker, L. Su, P. Carl, H. Zettl, J. He, K. Sill, R. Tangirala, T. Emrick, K. Littrell, P. Thiyagarajan, D. Cookson, 
A. Fery, Q. Wang, T. P. Russell, Angew. Chem. Int. Ed. 2005, 44, 2420.

[26] B. Kowalczyk, M. M. Apodaca, H. Nakanishi, S. K. Smoukov, B. A. Grzybowski, Small 2009, 5, 1970.

[27] H. Schlicke, J. H. Schröder, M. Trebbin, A. Petrov, M. Ijeh, H. Weller, T. Vossmeyer, Nanotechnology 2011, 22, 305303.

[28] K. Hirai, B. Yeom, S.-H. Chang, H. Chi, J. F. Mansfield, B. Lee, S. Lee, C. Uher, N. A. Kotov, Angew. Chem. Int. Ed. 2015, 54, 8966.

[29] A. A. Mamedov, N. A. Kotov, Langmuir 2000, 16, 5530.

[30] W. Cheng, M. J. Campolongo, J. J. Cha, S. J. Tan, C. C. Umbach, D. A. Muller, D. Luo, Nat. Mater. 2009, 8, 519.

[31] J. Lee, G. Bhak, J.-H. Lee, W. Park, M. Lee, D. Lee, N. L. Jeon, D. H. Jeong, K. Char, S. R. Paik, Angew. Chem. Int. Ed. 2015, 54, 4571.

[32] T. P. Bigioni, X.-M. Lin, T. T. Nguyen, E. I. Corwin, T. A. Witten, H. M. Jaeger, Nat. Mater. 2006, 5, 265.

[33] R. Vendamme, S.-Y. Onoue, A. Nakao, T. Kunitake, Nat. Mater. 2006, 5, 494.

[34] A. Boker, J. He, T. Emrick, T. P. Russell, Soft Matter 2007, 3, 1231.

[35] S. Kinge, M. Crego-Calama, D. N. Reinhoudt, ChemPhysChem 2008, 9, 20.

[36] L. Hu, M. Chen, X. Fang, L. Wu, Chem. Soc. Rev. 2012, 41, 1350.

[37] K. Piradashvili, E. M. Alexandrino, F. R. Wurm, K. Landfester, Chem. Rev. 2016, 116, 2141.

[38] W. H. Binder, Angew. Chem. Int. Ed. 2005, 44, 5172.

[39] Z. Niu, J. He, T. P. Russell, Q. Wang, Angew. Chem. Int. Ed. 2010, 49, 10052.

[40] S. G. Booth, R. A. W. Dryfe, J. Phys. Chem. C 2015, 119, 23295.
[41] N. Glaser, D. J. Adams, A. Böker, G. Krausch, Langmuir 2006, 22, 5227.

[42] L. Isa, E. Amstad, K. Schwenke, E. Del Gado, P. Ilg, M. Kroger, E. Reimhult, Soft Matter 2011, 7, 7663.

[43] F. Reincke, S. G. Hickey, W. K. Kegel, D. Vanmaekelbergh, Angew. Chem. Int. Ed. 2004, 43, 458.

[44] B. Su, J.-P. Abid, D. J. Fermín, H. H. Girault, H. Hoffmannová, P. Krtil, Z. Samec, J. Am. Chem. Soc. 2004, 126, 915.

[45] N. Zheng, J. Fan, G. D. Stucky, J. Am. Chem. Soc. 2006, 128, 6550.

[46] Q. K. Ong, J. Reguera, P. J. Silva, M. Moglianetti, K. Harkness, M. Longobardi, K. S. Mali, C. Renner, S. De Feyter, F. Stellacci, ACS Nano 2013, 7, 8529.

[47] R. C. Van Lehn, M. Ricci, P. H. J. Silva, P. Andreozzi, J. Reguera, K. Voïtchovsky, F. Stellacci, A. Alexander-Katz, Nat. Commun. 2014, 5, 4482.

[48] P. C. Sadek, The HPLC Solvent Guide, 2nd ed., Wiley-Interscience, New York 2002.

[49] S. K. Ghosh, T. Pal, Chem. Rev. 2007, 107, 4797.

[50] K. Schwenke, L. Isa, E. Del Gado, Langmuir 2014, 30, 3069.

[51] C. J. Brinker, Sol-Gel Science: The Physics and Chemistry of Sol-Gel Processing, Academic Press, Boston 1990.

[52] R. R. Nair, P. Blake, A. N. Grigorenko, K. S. Novoselov, T. J. Booth, T. Stauber, N. M. R. Peres, A. K. Geim, Science 2008, 320, 1308.

[53] K. Y. Lee, M. Kim, J. Hahn, J. S. Suh, I. Lee, K. Kim, S. W. Han, Langmuir 2006, 22, 1817.

[54] S.-Y. Zhang, J.-W. Liu, C.-L. Zhang, S.-H. Yu, Nanoscale 2013, 5, 4223.

[55] Y. Wang, C. Duan, L. Peng, J. Liao, Sci. Rep. 2014, 4, 7565.

[56] Y. T. Park, A. Y. Ham, J. C. Grunlan, J. Phys. Chem. C 2010, 114, 6325. 